\title{
Radio definida por software y docencia en línea: experiencia en el Grado en Ingeniería Telemática*
}

\author{
Carmen Botella-Mascarell ${ }^{1}$, Sandra Roger ${ }^{1}$, Antonio Soriano-Asensi ${ }^{1}$, Joaquin \\ Perez $^{2}$, Jaume Segura-Garcia ${ }^{1}$, Santiago Felici-Castell ${ }^{1}$ y Enrique Navarro-Camba ${ }^{1}$ \\ ${ }^{1}$ Departament d'Informàtica, Universitat de València. Av. de la Universitat s/n, 46100 Burjassot \\ (SPAIN) \\ ${ }^{2}$ Departament d'Enginyeria Electrònica, Universitat de València. Av. de la Universitat s/n, 46100 \\ Burjassot (SPAIN)
}

\begin{abstract}
Devices based on software defined radio have been used in the last 6 years as motivating elements in the laboratory practices of digital communications courses at the Degree in Telematics Engineering of the University of Valencia. However, the development of these practices requires giving access to some hardware devices that are physically located in the laboratories of the School of Engineering. In this contribution, taking into account the current situation of hybrid learning and a possible transition to remote learning due to the COVID-19 pandemic, a system that allows the remote interaction with the software defined radio devices is proposed. This remote access system, in addition to allowing these devices to be used in laboratory practices even in a remote learning context, would allow students to access them outside laboratory practice hours. The results obtained in the Digital Communications Theory course demonstrate the high potential for motivation and increased academic involvement provided by the use of these devices, especially in contexts with low academic involvement, such as the 20202021 academic year.
\end{abstract}

Keywords: software defined radio, SDR, Digital Communications, remote learning.

\begin{abstract}
Resumen
Los dispositivos de radio definida por software se han utilizado en los últimos 6 años como elementos dinamizadores de las prácticas de laboratorio en las asignaturas de la materia de comunicaciones digitales del Grado en Ingeniería Telemática de la Universitat de València. Sin embargo, el desarrollo de estas prácticas requiere el acceso a unos
\end{abstract}

\footnotetext{
*Este trabajo ha sido financiado por el "Vicerectorat d'Ocupació i Programes Formatius" de la Universitat de València a través del proyecto UV-SFPIE_PID-1353656. Sandra Roger agradece la finaciación recibida a través del contrato postdoctoral RYC-2017-22101 y el proyecto GV/2020/046.
} 
dispositivos hardware que están localizados físicamente en los laboratorios de la Escuela Técnica Superior de Ingeniería. En esta contribución, atendiendo a la actual situación de docencia semipresencial y de un posible paso a docencia en línea debido a la pandemia de COVID-19, se plantea establecer un sistema que permita la interacción remota con los dispositivos de radio definida por software. Este sistema de acceso remoto, además de permitir que se sigan utilizando estos dispositivos en las prácticas de laboratorio aún en un contexto de docencia en línea, permitiría el acceso del alumnado a ellos fuera del horario de prácticas de laboratorio. Los resultados obtenidos en la asignatura de Teoría de la Comunicación demuestran el alto potencial de motivación y de aumento de la implicación académica que presentan estos dispositivos, especialmente en contextos con una implicación académica baja, como es el curso académico 2020-2021.

Keywords: radio definida por software, SDR, Comunicaciones Digitales, docencia en línea.

\section{Introducción}

En este artículo se presenta el trabajo desarrollado en la asignatura Teoría de la Comunicación (TC) para adaptar las prácticas de laboratorio a posibles situaciones de docencia en línea en el curso 2020-2021. La asignatura TC es una asignatura que se imparte en el primer cuatrimestre de tercer curso en el Grado en Ingeniería Telemática de la Universitat de València (GIT-UV), en la Escuela Técnica Superior de Ingeniería (ETSE-UV). Pertenece a la materia de comunicaciones digitales (24 ECTS), consta de 6 ECTS y los contenidos desarrollan los distintos bloques que forman los sistemas de comunicaciones digitales: i) el transmisor (codificación de fuente, codificación de canal, modulación), ii) el canal (capacidad del canal y límites fundamentales), y iii) el receptor (cálculo de probabilidades de error).

En la materia de comunicaciones digitales se viene trabajando desde el curso 2015-2016 (Segura 2016; Soriano-Asensi 2019; Botella-Mascarell 2020) con el objetivo de introducir los dispositivos de radio definida por software (SDR, a partir de las siglas en inglés) en las prácticas de laboratorio, para acercar la docencia a sistemas de comunicaciones más realistas. Los dispositivos SDR (Mitola 1992) digitalizan la señal de radiofrecuencia y envían estas muestras a un ordenador, donde se realiza el procesado de la información a través de distintos interfaces como MATLAB, Labview o GNU Radio. El concepto de SDR ha evolucionado enormemente desde su aparición, tal y como recoge recientemente el trabajo de (Akeela 2018), donde se presentan las principales características y retos de estos dispositivos. En los últimos 10 años, el uso de estos dispositivos se ha demostrado también como una herramienta eficaz en la enseñanza relacionada con las telecomunicaciones (Bilén 2014; Stewart 2015; Wyglinski 2016; Xu 2018; Rice 2019). Estos dispositivos son muy versátiles y permiten implementar estándares de comunicaciones, como se puede ver en el trabajo de (Gil Jiménez 2017), al coincidir los bloques de los estándares de forma adecuada con los contenidos de las asignaturas de comunicaciones digitales. Además, existen implementaciones de bajo coste, lo que los hace accesibles a cursos con un volumen de alumnado elevado (Stewart 2015; Soriano-Asensi 2019).

En el caso de la materia de comunicaciones digitales en el GIT-UV, se empezó introduciendo el uso de las plataformas Universal Software Radio Peripheral (USRP). Estas plataformas son muy potentes, pero tienen un coste económico elevado, por lo que en el curso 2018-2019, se incorporaron 
soluciones de bajo coste como el RTL-SDR ${ }^{1}$ y de coste medio como el HackRF One ${ }^{2}$. La evaluación de esta metodología se realizó en un primer paso mediante encuestas a los estudiantes participantes (siempre voluntarios, y por tanto, con un sesgo a priori positivo), o bien cuantificando posibles mejoras en las notas de laboratorio o en las encuestas de satisfacción del alumnado realizadas por el sistema de control de calidad de la titulación (Segura 2016; Segura-García 2017). En un segundo paso, se incorporó el aprendizaje basado en proyectos (ABP), y se implementó una evaluación que cuantificara la actitud del estudiantado en el aula, para lo cual se empleó una encuesta basada en (Schaufeli y Bakker 2004; García-Ros 2018) para analizar el grado de implicación académica del estudiantado (Soriano-Asensi 2019). Con el objetivo de sistematizar la evaluación del impacto del uso de estos dispositivos, en el curso 2019-2020 se llevó a cabo una acción en la que las prácticas basadas en SDR se realizaban en un grupo experimental, manteniendo las sesiones simuladas estándar en dos grupos de control que trabajaban las sesiones simuladas (Botella-Mascarell 2020).

En el curso académico 2020-2021, y debido al impacto de la pandemia de COVID-19, la Comisión Académica de la Titulación (CAT) de Telecomunicaciones de la ETSE-UV estableció que las prácticas de laboratorio serían presenciales. Sin embargo, la posibilidad de que se decretara un confinamiento a lo largo de la asignatura motivó la puesta en marcha de la experiencia presentada en este artículo. Un problema que presentan las prácticas basadas en dispositivos hardware, es que dependen exclusivamente de elementos que físicamente están en la ETSE-UV, de manera que, frente a un confinamiento o paso a docencia en línea, el estudiantado no podría realizar la sesión de laboratorio. Se decidió por tanto habilitar un acceso remoto a los dispositivos, que además, sería de utilidad para aquellos estudiantes que no hubieran podido completar la práctica de laboratorio por alguna situación imprevista. De esta forma, este sistema de acceso remoto es de utilidad incluso en un contexto de docencia presencial.

El resto del artículo se organiza como sigue: en la sección 2 se enumeran los objetivos a conseguir en esta innovación docente y en la sección 3 se describe su desarrollo. La sección 4 presenta los resultados y su análisis, concluyendo el artículo en la sección 5.

\section{Objetivos}

Los objetivos generales de la acción de innovación se centran en la puesta en marcha del mecanismo que permite el uso remoto de los dispositivos SDR, para mantener la acción docente en situaciones de docencia en línea. Los tres objetivos generales son:

- Diseñar y desarrollar un sistema que permita el uso remoto de los dispositivos SDR.

- Establecer la metodología de uso de los dispositivos SDR en remoto y adaptar los materiales y recursos a la nueva situación.

- Evaluar el impacto de la metodología.

Los objetivos generales se pueden articular en los siguientes objetivos más específicos, que recogen los puntos a resolver:

\footnotetext{
${ }^{1}$ https://www.rtl-sdr.com

${ }^{2}$ https://greatscottgadgets.com/hackrf
} 
1. Implementar el sistema de acceso remoto a los dispositivos SDR para su uso durante las prácticas.

2. Establecer los mecanismos de seguridad adecuados para las comunicaciones SDR remotas.

3. Desarrollar los protocolos de acceso y uso de los dispositivos.

4. Adaptar los materiales a la nueva situación de docencia en línea.

5. Definir los instantes temporales para la recogida de evidencias.

6. Realizar los cuestionarios y evaluar los resultados obtenidos.

\section{Desarrollo de la innovación}

La asignatura Teoría de la Comunicación (primer cuatrimestre de tercer curso) forma parte de la materia de comunicaciones digitales del GIT-UV. El temario comprende los bloques de codificación de fuente (fuentes discretas y continuas), modulaciones digitales, capacidad de canal y codificación de canal. En el curso académico 2020-2021 la asignatura tiene 56 estudiantes matriculados, que se dividen en 3 grupos de laboratorio: AL1 (20 estudiantes), AL2 (18 estudiantes) y AL3 (18 estudiantes). El número final de estudiantes que realizó presencialmente los laboratorios fue: AL1 (18 estudiantes), AL2 (16 estudiantes) y AL3 (17 estudiantes). Todos los grupos de laboratorio realizan las sesiones en la misma franja horaria, en días distintos (martes, miércoles y viernes). Así mismo, los grupos AL2 y AL3 realizan las prácticas en idioma valenciano, siendo el AL1 en castellano. El alumnado puede realizar las sesiones de laboratorio de forma individual o en subgrupos de dos personas.

La Tabla 1 lista las 7 sesiones de laboratorio realizadas, todas de una duración de 2.5 horas. Las prácticas con SDR se realizan en las sesiones 5, 6 y 7. En la sesión 5, se utilizan los dispositivos RTL-SDR como receptores. Los bloques de recepción a evaluar (modulación QPSK, pulso de coseno alzado, cálculo de probabilidades de error) se construyen en el entorno Simulink de MATLAB. En la sesión 6 se utiliza el entorno GNU Radio para evaluar las prestaciones de códigos bloque en función de parámetros como la modulación (BPSK, QPSK) y la potencia de ruido presente en el sistema. En la sesión 7 se implementa un sistema completo de comunicaciones digitales, configurando el alumnado los receptores con los RTL-SDR y el profesorado el transmisor con una USRP. La práctica permite ajustar aspectos como la sincronización de los RTL-SDR o la orientación óptima de las antenas receptoras.

La evaluación de las sesiones de laboratorio forma parte de la evaluación continua de la asignatura, representando un $15 \%$ de la nota final de la asignatura en ambas convocatorias. Tras cada sesión de laboratorio, los grupos disponen de una semana de plazo para adjuntar en el Moodle de la asignatura o bien el script de MATLAB realizado, o los esquemáticos de Simulink o GNU Radio, incluyendo pequeñas memorias en aquellas sesiones en las que hay que desarrollar alguna pregunta corta.

Para desarrollar la innovación de forma adecuada, el primer paso fue la definición y la puesta en marcha de un sistema que permitiera acceder a los dispositivos SDR sin estar el alumnado presencialmente en la ETSE-UV. Hay que destacar que esta metodología requiere que al menos una persona (profesorado, personal de apoyo de los laboratorios) esté presente en la ETSE-UV para realizar la conexión de los dispositivos a los ordenadores del aula durante la sesión de laboratorio, 
Tabla 1: Sesiones de laboratorio y contenido. Elementos SDR utilizados

\author{
Sesión / Contenido \\ $\mathbf{1}$ / Probabilidad y entropía \\ 2 / Algoritmo de Huffman \\ 3 / Codificación aritmética \\ 4 / Conversión analógico-digital. Cuantificación \\ 5 / Sistema de transmisión digital: recepción (RTL-SDR, Simulink) \\ 6 / Códigos bloque, código de repetición (GNU Radio) \\ 7/ Sistema de transmisión digital: transmisión y recepción (RTL-SDR, USRP, Simulink)
}

ya que los RTL-SDR son dispositivos muy sencillos que pueden presentar problemas de disipación de potencia si permanecen durante mucho tiempo conectados. La Figura 1 muestra el sistema de acceso remoto establecido para las prácticas de laboratorio, donde una herramienta fundamental es el uso del programa Microsoft Teams, que es el utilizado para realizar la docencia en línea. Por sencillez, se decidió que el acceso remoto fuese mediante herramientas de conexión a escritorio remoto, de manera que el alumnado pudiera acceder de forma remota al ordenador que físicamente está conectado al dispositivo. Este mecanismo presenta el problema de que no se puede habilitar la conexión por escritorio remoto en los ordenadores de las aulas de laboratorio por motivos de seguridad de los equipos. Por este motivo, se montaron 4 equipos informáticos para uso exclusivo en las prácticas de aquellas asignaturas que utilizaran SDR.

Siguiendo las indicaciones de seguridad del servicio de informática, los equipos se configuraron para admitir únicamente conexiones en remoto de equipos cuya IP quedara dentro del rango de direcciones IP asignadas por la Universitat de València. Por este motivo, para establecer la conexión a escritorio remoto, el alumnado debía realizar previamente una red privada virtual (virtual private network (VPN)), obteniendo así una IP adecuada. Puesto que únicamente había 4 equipos informáticos para acceder a los dispositivos SDR y el número de subgrupos dentro de un grupo de laboratorio podía llegar a 9, fue necesario establecer un protocolo de acceso y realizar una adaptación de los guiones de las sesiones de laboratorio. Las sesiones que se impartieron en línea contaban con el apoyo de la herramienta Microsoft Teams, que permite videollamadas, chats grupales, compartir pantalla, etc. Para acceder a los equipos en remoto, se establecieron turnos de 4 subgrupos para realizar las conexiones a escritorio remoto. Cuando a un subgrupo le tocaba el turno de conexión, uno de sus componentes realizaba la VPN y establecía la conexión en remoto. A continuación, utilizando un chat grupal de Microsoft Teams, compartía pantalla con el otro componente de su subgrupo, de manera que todos los integrantes del subgrupo tenían acceso al dispositivo SDR en remoto. Además, el chat grupal permite agregar al profesorado, de forma que se pueden resolver dudas o problemas de forma inmediata. Para poder establecer los turnos, los guiones de las sesiones se modificaron ligeramente para evitar que algunos subgrupos experimentaran tiempos de espera.

La evaluación de la acción de innovación se programó en dos instantes temporales: al inicio de la sesión 5 (primera sesión que utiliza dispositivos SDR), y al final de la sesión 7 . Hay que destacar que el estudiantado realizó el cuestionario de forma voluntaria y anónima a través de un recurso del Moodle de la asignatura. 


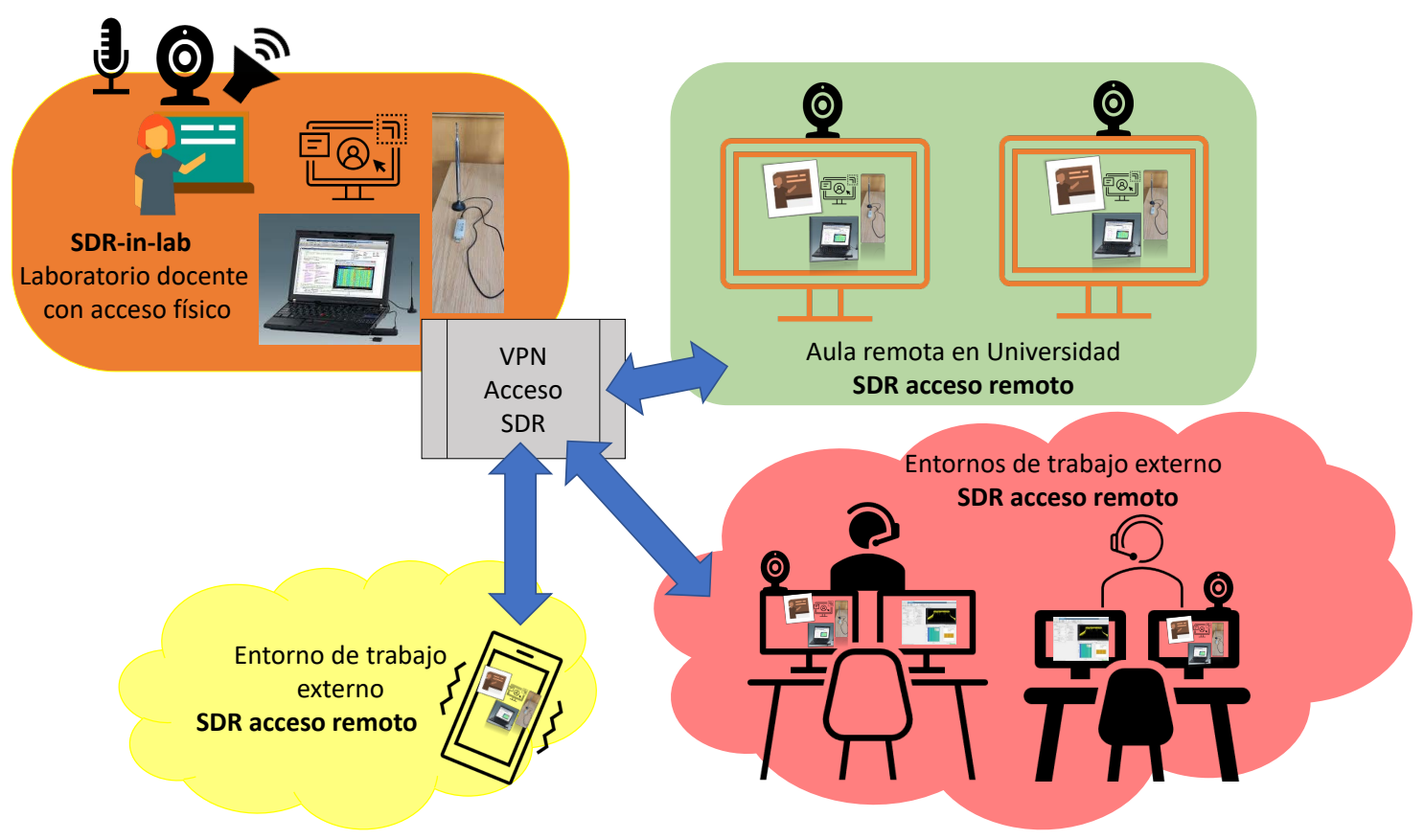

Fig. 1: Sistema de acceso remoto. Comprende un laboratorio docente donde se encuentran físicamente los dispositivos SDR. El alumnado puede acceder de forma remota estableciendo una conexión VPN (desde aulas espejo en la ETSE-UV o desde fuera de la ETSE-UV)

\section{Resultados}

Los dispositivos SDR se utilizan en la asignatura como elementos motivadores y dinamizadores en la enseñanza de las comunicaciones digitales. Por ese motivo, para evaluar el impacto del uso de estos dispositivos, se ha utilizado una adaptación de un cuestionario que evalúa el compromiso en el trabajo (Schaufeli y Bakker 2004; García-Ros 2018; Soriano-Asensi 2019; Botella-Mascarell 2020). El motivo de seleccionar este cuestionario fue la necesidad de valorar de forma objetiva la implicación académica del alumnado, puesto que en experiencias previas se utilizaron como elementos de evaluación las calificaciones obtenidas en las prácticas de laboratorio, o los resultados de las encuestas de evaluación de calidad de la enseñanza (Segura 2016; Segura-García 2017). Existen otras metodologías de evaluación como la rúbrica propuesta en (Rice 2019), que requiere que todas las sesiones de laboratorio utilicen dispositivos SDR. En nuestro caso, el estudiantado valoró 10 preguntas utilizando una escala Likert entre 1 (nunca/casi nunca) y 5 (casi siempre/siempre): 
La siguiente encuesta se realiza dentro del marco de un proyecto de innovación docente financiado para el curso 2020-2021. Las respuestas son anónimas, y se utilizan con el objetivo de mejorar la docencia de las asignaturas del bloque de Comunicaciones Digitales.

Tenga en cuenta que las preguntas de la encuesta se refieren a las sesiones de prácticas de laboratorio. Para responder, destaque hasta qué punto las afirmaciones de las preguntas describen su situación en este momento del curso. Responda con sinceridad, utilizando una escala entre 1 (nunca/casi nunca) y 5 (casi siempre/siempre).

- Q1 - En el laboratorio me siento lleno/llena de energía;

- Q2 - Pienso que las prácticas son relevantes y significativas;

- Q3 - El tiempo me parece que "vuela" mientras realizo las prácticas;

- Q4 - Me siento con gran fuerza y vigor durante las prácticas;

- Q5 - Me entusiasman las prácticas que se nos proponen;

- Q6 - Mientras realizo las prácticas me olvido de todas las cosas que pasan a mi alrededor;

- $\mathbf{Q 7}$ - El trabajo en el laboratorio me resulta ilusionante;

- $Q 8$ - Tengo ganas de ir a clase cuando llego a la Universidad (si se encuentra en un periodo de clase on-line, cambie la pregunta por: Tengo ganas de participar en la clase);

- $\mathbf{Q 9}$ - Me satisface trabajar con intensidad en el laboratorio;

- $\mathbf{Q 1 0}$ - En general, me siento muy satisfecho/a con las actividades que se nos proponen en el laboratorio.

Las preguntas del cuestionario tienen como objetivo evaluar tres dimensiones de la implicación académica: i) energía, relacionada con la capacidad de resiliencia del estudiantado frente a la resolución de problemas (Q1, Q4, Q8); ii) absorción, relacionada con la capacidad del estudiantado para concentrarse en aquellas tareas que está realizando (Q3, Q6, Q9); y iii) dedicación, relacionada con la percepción del estudiantado sobre la relevancia de las actividades que realiza (Q2, Q5, Q7). La satisfacción general se evalúa mediante la pregunta Q10. El cuestionario recoge en la pregunta Q7 una pequeña variación para adaptarse a las situaciones en las que pudiera darse un periodo de clase en línea.

De las tres sesiones planteadas para utilizar dispositivos SDR, las sesiones 5 y 6 se realizaron en línea utilizando el mecanismo descrito, mientras que la sesión 7 se realizó de forma presencial. El número de encuestas recogidas en el muestreo previo fueron de 16, 14 y 16, por cada grupo, respectivamente, mientras que en el muestreo posterior se recogieron 16, 13 y 14, respectivamente. La Tabla 2 recoge los valores promedio obtenidos en cada pregunta en los dos instantes temporales, así como la diferencia entre ambos valores, mientras que la tabla Tabla 3 muestra los resultados promedio agregados por cada una de las dimensiones (energía, absorción y dedicación). Los resultados mostrados en la Tabla 2 y la Tabla 3 muestran, por un lado, que la implicación del estudiantado en el muestreo previo era muy baja, probablemente influenciada por el contexto asociado a la pandemia de COVID-19, como indican los resultados obtenidos en las encuestas llevadas a cabo con el cuestionario Covid19impactsurvey.org (http://argos.gva.es/es/encuestas/covid-19) 
del grupo de trabajo "Data Science for COVID-19 TaskForce". Los resultados de este cuestionario muestran que las personas jóvenes son las que se están viendo anímicamente más afectadas por la situación asociada a la pandemia. Por otro lado, los resultados posteriores muestran que la innovación ha tenido un impacto muy positivo, superando experiencias previas como la presentada en (Botella-Mascarell 2020), correspondiente a un curso académico sin incidencias, demostrando la capacidad de motivación que presenta el uso de dispositivos SDR y justificando el desarrollo de soluciones que permitan utilizarlos dentro de la docencia en línea.

Tabla 2: Valores promedio obtenidos en cada una de las preguntas del cuestionario, antes y después de realizar las prácticas basadas en SDR. Diferencia entre ambos valores

\begin{tabular}{lcccccccccc}
\hline & Q1 & Q2 & Q3 & Q4 & Q5 & Q6 & Q7 & Q8 & Q9 & Q10 \\
\hline Previo & 2.91 & 3.52 & 3.11 & 2.78 & 2.7 & 2.67 & 2.87 & 2.85 & 3.2 & 3.17 \\
Posterior & 3.67 & 4.02 & 3.7 & 3.49 & 3.58 & 3.23 & 3.47 & 3.09 & 3.7 & 3.79 \\
Diferencia & 0.76 & 0.50 & 0.59 & 0.71 & 0.89 & 0.56 & 0.60 & 0.25 & 0.50 & 0.62 \\
\hline
\end{tabular}

Tabla 3: Variación de los valores promedio de energía, absorción y dedicación del estudiantado

\begin{tabular}{llll}
\hline & Energía & Absorción & Dedicación \\
\hline Previo & 2.85 & 2.99 & 3.03 \\
Posterior & 3.42 & 3.54 & 3.69 \\
Diferencia & 0.57 & 0.55 & 0.66 \\
\hline
\end{tabular}

La mayor diferencia en la Tabla 2 se encuentra en la pregunta Q5, mientras que el menor incremento se encuentra en la pregunta Q8. En el muestreo previo, se observa que la pregunta que obtiene un menor valor es la Q6, mientras que en el muestreo posterior es la pregunta Q8 la que obtiene un menor valor promedio. Por otro lado, la pregunta que obtiene un mayor valor en el muestreo previo es la Q2, manteniéndose también como la pregunta que obtiene un mayor valor promedio en el muestreo posterior. Es importante observar que la pregunta asociada a la satisfacción general, la Q10, se posiciona, tanto en el muestreo previo como en el posterior, como una de las preguntas que mejores resultados obtiene. La Tabla 3 indica que la dimensión de dedicación es la que mayor incremento obtiene en valor promedio al comparar el muestreo previo con el posterior. Además, es la dimensión que presenta un mayor valor promedio tanto en el muestreo previo como en el posterior. La dimensión de energía es la que presenta menores valores promedio en los dos muestreos temporales, coincidiendo con lo que se observa en el cuestionario Covid19impactsurvey.org.

Para poder analizar los resultados con más detalle, se muestran en la Figura 2 las probabilidades de los valores de la escala Likert obtenidas de forma agregada para cada dimensión de la implicación académica, en el muestreo previo y en el muestreo posterior. En la figura se observa cómo en las 

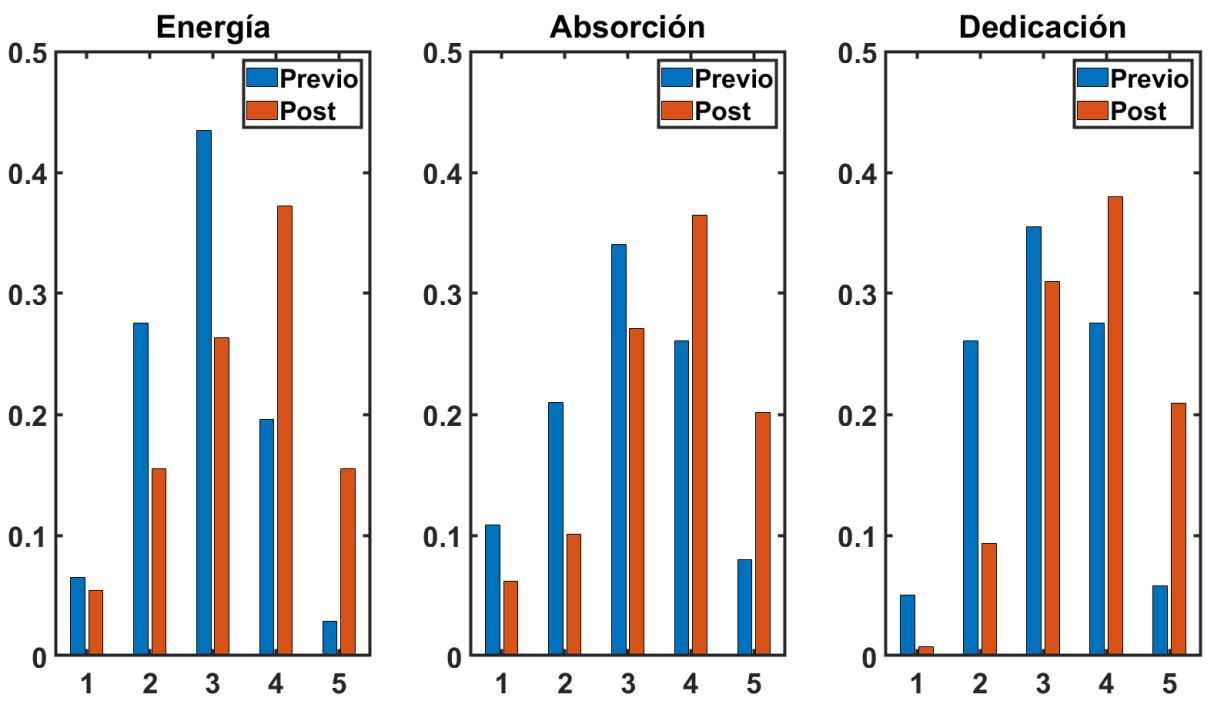

Fig. 2: Probabilidad de los valores de la escala Likert obtenida de forma agregada para las dimensiones de energía, absorción y dedicación en el muestreo previo y posterior

tres dimensiones evaluadas se produce un incremento importante en las valoraciones más altas, siendo especialmente notable el incremento de la valoración 5 - casi siempre/siempre. Respecto a las valoraciones más bajas, es la dimensión de dedicación la que en el muestreo posterior consigue disminuir de forma considerable los resultados asociados a la valoración más baja 1 - nunca / casi nunca.

Como se ha observado en trabajos anteriores como el presentado en (Botella-Mascarell 2020), la metodología de uso de dispositivos SDR supone un mayor esfuerzo para el profesorado implicado. En la experiencia presentada en este artículo, además, ha sido necesario organizar un sistema de acceso en remoto a los dispositivos SDR para poder cubrir las situaciones de docencia en línea. Sin embargo, los resultados obtenidos han sido muy positivos en las tres dimensiones evaluadas de implicación académica. Para corroborar este efecto en otros aspectos más cuantitativos de la evaluación, se ha obtenido la nota media de las calificaciones obtenidas en las sesiones simuladas estándar (sesiones 1-2-3-4) y la nota media de las calificaciones obtenidas en las sesiones que utilizan dispositivos SDR (sesiones 5-6-7), siendo de 6.8 y de 8.2, respectivamente. Hay que destacar que este efecto sobre las notas medias de las calificaciones no se observó en la experiencia previa presentada en (Botella-Mascarell 2020), lo que demuestra el alto potencial motivador del uso de dispositivos SDR en las prácticas de laboratorio, especialmente en contextos donde la implicación académica inicial es baja, como ha sido el curso académico 2020-2021. 


\section{Conclusiones}

Los dispositivos de radio definida por software se han mostrado como elementos que permiten plantear prácticas de laboratorio más realistas y cercanas a los sistemas de comunicaciones actuales. $\mathrm{Al}$ mismo tiempo, las experiencias previas indican que presentan un alto potencial como elementos dinamizadores y motivadores en la docencia de las prácticas de laboratorio. En esta contribución, se ha presentado un sistema de acceso remoto que permite la realización de las prácticas de laboratorio en situaciones de docencia en línea. Los resultados obtenidos han sido muy positivos, superando los obtenidos en cursos académicos anteriores al presentar este curso académico un escenario de baja implicación del alumnado. El sistema presentado en esta contribución es también importante y de utilidad en un contexto de docencia exclusivamente presencial, ya que permite que el estudiantado acceda a estos dispositivos fuera del horario de las sesiones de laboratorio, permitiendo la realización de las prácticas en caso de situaciones imprevistas, así como dando acceso a otros estudiantes (trabajo fin de grado, trabajo fin de máster) que también puedan requerir el uso de estos dispositivos. En los próximos cursos académicos, se trabajará para extender esta metodología de trabajo y de evaluación de resultados en el resto de asignaturas de la materia.

\section{Referencias bibliográficas}

Akeela, R. y Col. (2018). "Software-defined Radios: Architecture, state-of-the-art, and challenges". En: Computer Communications 128, págs. 106-125.

Bilén, S.G. y Col. (mayo de 2014). "Software-defined radio: a new paradigm for integrated curriculum delivery". En: IEEE Communications Magazine 52.5, págs. 184-193.

Botella-Mascarell, C. y Col. (sep. de 2020). "Evaluación del impacto del uso de dispositivos de radio definida por software como herramienta docente en la materia de comunicaciones digitales". En: XXXV Simposium Nacional de la Unión Científica Internacional de Radio. Málaga (Spain).

García-Ros, R. y Col. (sep. de 2018). "The schoolwork engagement inventory: factorial structure, measurement invariance by gender and educational level, and convergent validity in secondary education (12-18) years." En: Journal of Psychoeducational Assessment 36.6, págs. 588-603.

Gil Jiménez, V.P. y Col. (mayo de 2017). "Learning mobile communications standards through flexible software defined radio base stations". En: IEEE Communications Magazine 55.5, págs. 116-123.

Mitola, J. (mayo de 1992). "Software radios-survey, critical evaluation and future directions". En: Proc. IEEE National Telesystems Conference, (NTC'92). Washington DC, USA, págs. 15-23.

Rice, M. y Col. (nov. de 2019). "Teaching communications with SDRs: making it real for students". En: IEEE Communications Magazine 57.11, págs. 14-19.

Schaufeli, W. y A. Bakker (dic. de 2004). UWES-Utrecht Work Engagement Scale: Test manual. Department of Psychology, Utrecht University, Utrecht, The Netherlands. 
C. Botella-Mascarell, S. Roger, A. Soriano-Asensi, J. Perez, J. Segura-Garcia, S. Felici-Castell y E. Navarro-Camba

Segura, J. y Col. (jul. de 2016). "Innovación en docencia de sistemas de comunicación en el Grado de Ingeniería Telemática de la UVEG". En: IN-RED 2016. II Congreso de Innovación Educativa y Docencia en Red. Valencia (Spain).

Segura-García, J. y Col. (sep. de 2017). "Uso de Software-Defined Radio en la enseñanza de sistemas de comunicaciones". En: XIII Jornadas de Ingeniería telemática (JITEL). Valencia (Spain).

Soriano-Asensi, A. y Col. (jul. de 2019). "Aprendizaje basado en proyectos en los laboratorios de comunicaciones digitales". En: IN-RED 2019. V Congreso de Innovación Educativa y Docencia en Red. Valencia (Spain).

Stewart, R.W. y Col. (sep. de 2015). "A low-cost desktop software defined radio design environment using MATLAB, simulink, and the RTL-SDR". En: IEEE Communications Magazine 53.9, págs. 64-71.

Wyglinski, A.M. y Col. (ene. de 2016). "Revolutionizing software defined radio: case studies in hardware, software, and education". En: IEEE Communications Magazine 54.1, págs. 68-75.

Xu, Z. y Col. (dic. de 2018). "Design and Evaluation of SDR-based Labs for Learning Principles of Communications". En: Proc. IEEE International Conference on Teaching, Assessment, and Learning for Engineering (TALE). Wollongong, NSW, Australia, págs. 1049-1052. 